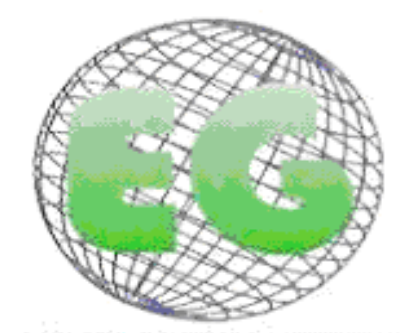

ISSN 1695-6141 N 27

\title{
El papel de los síndromes culturales y los remedios tradicionales mexicanos en la promoción de salud de los niños
}

The role of Culture-bound syndromes and Mexican Folk Healing in Child Health Promotions

\author{
${ }^{*}$ Gallagher, MR., ${ }^{* *}$ Rehm, RS.
}

*PhD, RN. Assistant Professor University of Texas Health Science Center-School of Nursing Houston, TX. E-mail: Martina.R.Gallagher@uth.tmc.edu ${ }^{* * P h D, ~ R N, ~ F A A N . ~ A s s o c i a t e ~ P r o f e s s o r ~ U n i v e r s i t y ~ o f ~}$ California at San Francisco. USA.

Palabras clave: Remedios tradicionales; terapias alternativas complementarias; promoción de la salud; mexicano; materno/infantil.

Keywords: Folk Healing; Complementary Alternative Therapies; Health Promotion; Mexican; Maternal/Child

\section{RESUMEN}

Objetivo: Describir el contexto en el que los síndromes culturales de las madres de ascendencia mexicana creían que afectaban a la salud infantil y describir cómo ellas mejoraban la salud cuando estos síndromes afectaban a sus hijos.

Diseño: Los resultados de este estudio se basan en una amplia investigación que se centró en la promoción de la salud y las prácticas de protección usadas por las madres de ascendencia mexicana en la ciudad de Texas. Se seleccionó un diseño naturalista, mediante las técnicas de entrevistas etnográficas de Spradley y observaciones de los participantes, para explorar y describir la promoción de la salud infantil y las prácticas de protección, incluyendo los síndromes culturales usados por las madres de origen mexicano.

Método: La recopilación de los datos consistió en 21 entrevistas etnográficas mejoradas por el enfoque de las observaciones en hogares con 9 madres de habla hispana.

Resultados: Para este grupo de mujeres, los síndromes culturales del "empacho", "mal de ojo" y "susto" podían provocar trastornos alimenticios y del sueño, causando un desajuste en el bienestar del niño. Además, las participantes creían que ellas tenían que ser conscientes de los síndromes culturales que afectaban a la salud de sus hijos y cuidar aquellos síndromes usando remedios tradicionales para promocionar y proteger la salud de sus niños.

Conclusión: Los resultados de este estudio ofrecen una descripción en profundidad de los síndromes culturales y los remedios tradicionales que las madres de ascendencia mexicana usaban para promocionar y proteger la salud de sus niños. Este conocimiento ofrece un marco para que los 
profesionales de la salud lo usen cuando trabajen con madres de ascendencia mexicana que pueden estar usando este tipo de medios para cuidar a sus hijos.

\section{ABSTRACT}

Purpose: To describe the context in which culture-bound syndromes that mothers of Mexican descent believed affected child wellness and describe how they restored health when these syndromes affected their children.

Design: The findings of this come from a larger study that focused on the health promotion and protection practices used by mothers of Mexican descent in urban Texas A naturalistic design, using Spradley's ethnographic interview techniques and participant observations, was selected to explore and describe the child health promotion and protection practices, including culture-bound syndromes, used by mothers of Mexican origin.

Method: Data collection consisted of 21 ethnographic interviews enhanced by focused home observations with nine Spanish speaking mothers.

Results: To these group of mothers, the culture-bound syndromes of empacho, fright and evil eye could affect children's eating and sleep patterns, thereby causing an imbalance in a child's wellbeing. Therefore the participants believed that they had be mindful of culture-bound syndromes that affected their children's health and take care of those syndromes by using folk remedies to restore balance in their children's' wellbeing.

Conclusion: The findings of this study provide an in-depth description of culture-bound syndromes and the folk remedies which mother of Mexican descent used to promote and protect the health of their preschool children. This knowledge provides a framework for healthcare professionals to use when working with mothers of Mexican descent who may be using folk healing to promote and protect the health of their children.

\section{INTRODUCCIÓN}

Los latinos es uno de los mayores grupos de minorías étnicas en los Estados Unidos, siendo estas personas principalmente de ascendencia mexicana ${ }^{(1)}$. Durante siglos, personas de ascendencia mexicana han usado los remedios tradicionales para resolver los síndromes culturales ${ }^{(2)}$. Los síndromes culturales son enfermedades y dolencias restringidas a un grupo o cultura en particular ${ }^{(3)}$. Unos pocos estudios habían descrito los síndromes culturales y los remedios tradicionales usados por las madres de ascendencia mexicana con sus niños ${ }^{(4,5)}$. Sin embargo, estos estudios carecían de un razonamiento significativo o contextualizado sobre los remedios tradicionales usados por las madres con sus hijos.

Para que el equipo de salud pueda fomentar las interacciones culturales con las familias de ascendencia mexicana, es importante comprender la perspectiva significativa de los síndromes culturales que afectan a los niños y el razonamiento usado para los remedios tradicionales. Asimismo es importante entender qué remedios son usados por las madres mexicanas para ofrecer un cuidado seguro y apropiado por parte de los enfermeros, y controlar cualquier factor de riesgo que pueda estar presente si los remedios no son seguros o muestran cualquier contraindicación al cuidado médico.

\section{Síndromes culturales en la cultura mexicana}

En la cultura mexicana, los síndromes culturales que afectan a la salud de los niños son el "susto", "el empacho" y el "mal de ojo" (4,5). Risser y Mazur encontraron en un estudio en Houston, Texas, que el $70 \%$ de los padres de ascendencia mexicana creían en el "mal de ojo", el $64 \%$ creían en el "empacho" y el $37 \%$ creían en el "susto" (5). Mikhail también encontró una muestra similar a la que hay en el presente estudio en la que los participantes creían en el "empacho", el "susto" y en el "mal de ojo" (4). El "susto" es una dolencia tradicional de la que 
se piensa que provoca trastornos alimenticios y del sueño en los niños. Del "empacho" se cree que es provocado por un pedazo de masa cruda que se adhiere a la pared del estómago o porque el estómago se adhiere a sí mismo. Los niños que sufren "empacho" normalmente pierden el apetito, tienen náuseas y en ocasiones, diarrea. El "mal de ojo" se produce cuando alguien mira al niño intensamente, normalmente con admiración. Con frecuencia, cuando los niños cogen el mal de ojo, no descansan, lloran sin razón y no pueden dormir.

A pesar de nuestro conocimiento de los síndromes culturales y sus remedios, no está claro cómo las madres de ascendencia mexicana contextualizan los marcos de estas enfermedades y remedios. El objetivo de este artículo es describir los síndromes culturales que las madres de ascendencia mexicana creían que afectaban a la salud infantil y describir cómo ellas los curaban cuando estos síndromes afectaban a sus hijos. Además, describimos el proceso en el que estas prácticas ocurren. Los resultados provienen de un amplio estudio que se centró en la promoción de la salud y en las prácticas de protección usadas por las madres de ascendencia mexicana en la ciudad de Texas ${ }^{(6)}$. En este artículo también se presentan los resultados relacionados con los síndromes culturales que afectaban la salud infantil y las prácticas maternas para protegerla.

\section{MÉTODO}

\section{Diseño del estudio}

Se escogió un estudio naturalista, usando las técnicas de entrevistas etnográficas de Spradley y las observaciones a los participantes, para explorar y describir la promoción de la salud infantil y las prácticas de protección, incluyendo los síndromes culturales usados por las madres de origen mexicano. A continuación, se presenta una descripción detallada del estudio y de los métodos empleados ${ }^{(6)}$.

\section{Muestras}

La universidad patrocinadora obtuvo la aprobación de los participantes. Su consentimiento se consiguió antes de la recopilación de datos. La contratación de los participantes sucedió durante las actividades de la comunidad. Las madres que se autoidentificaban como de ascendencia mexicana, tenían 18 años de edad o más, tenían al menos dos hijos, con uno menor de 5 años, y que hablaban inglés o español, se incluyeron en el estudio. Se excluyó a aquellas madres que tenían un hijo menor de 5 años que había sido diagnosticado de alguna enfermedad crónica, retrasos del crecimiento o cualquier discapacidad física o mental.

\section{Entorno}

El marco del estudio fue el centro de una comunidad religiosa que sirve a una amplia comunidad de latinos de la gran ciudad en Texas. Algunos servicios ofrecidos por el centro son bancos de alimentos, clases de educación física, clases de paternidad e inglés como segunda lengua.

\section{Recopilación de datos}

Los datos se recopilaron a través de entrevistas etnográficas semi-estructuradas, las observaciones a los participantes se registraron como notas de campo, una hoja de diseño investigador de datos demográficos y una medida de aculturación como descriptor demográfico. La recopilación de datos tuvo lugar entre Diciembre de 2004 y Julio de 2005. 


\section{Entrevistas etnográficas}

El guión de la entrevista contenía preguntas descriptivas, estructurales y de contraste ${ }^{(7)}$ para obtener información sobre la promoción de la salud y las prácticas de protección que los participantes usaban con sus niños. Las entrevistas tuvieron lugar en 3 momentos, siendo entrevistada cada madre al menos en 2 ocasiones. Se llevaron a cabo un total de 21 entrevistas con 9 participantes. Las preguntas iniciales descriptivas fueron "¿Qué tipo de cosas haces para proteger la salud de tu hijo?”, “¿Qué tipo de cosas haces para evitar que tu hijo enferme?” y “¿Qué tipo de cosas haces para prevenir que tu hijo se hiera físicamente?”.

Tras un análisis de dominio, las posteriores entrevistas incluyeron preguntas estructurales. Por ejemplo, las madres explicaron que una forma de proteger a sus hijos del "mal de ojo" era poniéndole un amuleto al niño. Se hicieron preguntas estructurales tales como "¿Qué cosas haces para poder proteger a tu hijo del "mal de ojo?", y preguntas de contraste como por ejemplo: “¿cuál es la diferencia entre el "mal de ojo” y el "susto"?” para conseguir una mejor comprensión de cada síndrome cultural de manera individual y de las prácticas de promoción de la salud.

\section{Observación de los participantes}

Para generar confianza con los participantes, el primer autor pasó 70.5 horas en las observaciones a los participantes en varias actividades en el entorno del estudio. Estas actividades fueron clases de paternidad, salsa y aerobic, voluntariado en bancos de alimentos y reuniones de las familias de la comunidad por las noches. Las observaciones a los participantes y las repetidas entrevistas con la oportunidad de discutir el análisis preliminar mejoró el compromiso prolongado que ayudó a establecer credibilidad y validez a los datos ${ }^{\left({ }^{(8)}\right.}$.

\section{Demografía}

En la primera entrevista se obtuvo la información demográfica. Los datos demográficos incluyeron la edad de los participantes, educación, nivel laboral, estado civil, número de niños en casa, número y edades de los niños en educación infantil, número de las personas que vivían en la casa, tiempo vivido en los Estados Unidos, lenguas habladas y creencias religiosas.

\section{Aculturación}

La aculturación materna fue medida por el ARSMA-II ${ }^{(9)}$. El ARSMA-II evalúa las actitudes y comportamientos con respecto a las etnias mexicana e inglesa. Cuellar y al. informaron sobre las inconsistencias internas que tenían las sub-escalas del ARSMA-II que se registraban desde .68 hasta .96. La fiabilidad de los test-retest de las sub-escalas variaba desde 72 . hasta 96., y la validez concurrente es .89. Las puntuaciones de aculturación se analizaron con el programa ARSMA-II ${ }^{(9)}$ y se transfirieron al SPSS para la gestión de los datos.

\section{Gestión de los datos y análisis}

Las entrevistas se llevaron a cabo en español y fueron grabadas en audio y transcritas verbalmente. La precisión en la transcripción se comprobó por el primer autor y los dos enfermeros que estaban familiarizados con el tema y hablaban en español de una manera fluida. Los datos en formato de texto fueron administrados a través del programa Atlas ti, versión 4.2. Los datos demográficos se transfirieron al SPSS para gestionarlos. Para preservar el significado cultural en los datos, el análisis etnográfico y la documentación inicial 
de los resultados se hizo en español. Los resultados no se tradujeron al inglés hasta el informe final.

Las transcripciones se analizaron después de que se alcanzase la redundancia informativa con cada ola de entrevistas. Los análisis se dieron en cuatro niveles: (a) análisis de dominio, (b) análisis taxonómico (un estudio más a fondo de las estructuras internas de cada dominio), (c) análisis componencial (una búsqueda de los atributos que marcan diferencias entre los símbolos en un dominio) y (d) análisis temático (una búsqueda de las relaciones entre los dominios y sus relaciones con el conjunto). Los temas culturales se obtuvieron de varios análisis. Estos temas culturales se discutían al final de la entrevista con todos los participantes.

\section{RESULTADOS}

\section{Características de la muestra}

La muestra consistió en nueve madres de ascendencia mexicana. La redundancia informativa se alcanzó con todos los participantes. La edad de los participantes oscilaba desde los 24 hasta los 40 años, con una media de $32.6(\sigma=4.8)$. Seis de las participantes estaban casadas. Dos participantes estaban separadas y una estaba viviendo con su pareja de hecho. Dos de las nueve participantes vivía con dos o más adultos en sus casas, además de sus esposos. Tan solo dos participantes enviaban a sus hijos a la guardería financiada por la comunidad. El número de años que las participantes residieron en los Estados Unidos variaba desde 1 hasta 24 años, con una media de 10.7 ( $\sigma=8.9$ ). Tres de las nueve participantes eran bilingües; sin embargo, ellas preferían comunicarse en español. Basados en los resultados del ARSMA, este grupo de madres estaba orientado a la cultura mexicana y no se había adaptado a la dominante cultura estadounidense. Tan solo una participante no trabajaba. El resto de participantes eran autónomas o trabajaban a media jornada como amas de llaves en hogares y negocios de la comunidad. Todos los hijos de las participantes de infantil estaban matriculados en Medicaid, lo que indicaba que los ingresos de las familias de las participantes no superaban el $133 \%$ del nivel federal de pobreza ${ }^{(10)}$. Ocho de las nueve participantes eran católicas practicantes. Una participante afirmó que ella no simpatizaba con ninguna creencia religiosa.

\section{Síndromes culturales y su efecto en la salud de los niños}

Las participantes en este estudio creían en los síndromes culturales del "empacho", "mal de ojo" y del "susto". Los participantes en este estudio enmarcaban los síndromes culturales en dos contextos diferentes: aquellos que afectaban a los patrones alimenticios y del apetito, y aquellos que afectaban a los patrones del sueño. Los síndromes culturales que afectaban a los patrones alimenticios y del apetito eran el "empacho" y el "susto". Los síndromes culturales que afectaban al sueño de los niños eran el "susto" y el "mal de ojo".

\section{El empacho y su efecto en la comida}

Una participante describió cómo el empacho afectaba a la alimentación de los niños:

Porque su estómago no está funcionando como debería...la comida que él come se queda ahí [en el estómago]... y eso no permite... que haga una digestión normal. Entonces, cuando su estómago está demasiado pesado, no tiene apetito. Yo intento darle [comida] y le ofrezco, pero no quiere comer nada porque no siente que tenga 
hambre. Entonces es cuando tú tienes que sacárselo [haciéndole fricción] de tal forma que su estómago vuelva a funcionar.

Las madres normalmente sabían que el "empacho" se iba cuando los niños volvían a comer.

Las madres explicaban que ellas probablemente podían prevenir el "empacho" de los niños asegurándose de que no comieran comida que no estaba bien cocinada. Una madre afirmó, "quizás podrías prevenirlo si no les dieras la harina de la masa de la tortilla cruda". También se creía que el "empacho" se causaba cuando los niños comían algo que no les sentaba bien a sus estómagos. Todas las participantes creían que los productos con harina que no estaban del todo cocinados se pegarían en los estómagos de sus hijos impidiendo la digestión. Ésta falta de digestión provocaría que los niños dejasen de comer.

El "empacho" normalmente se ha tratado masajeando el estómago y la espalda de los niños. Las madres llamaban a este masaje que cura el "empacho" una "sobada". Una participante afirmó que el empacho se curaba mediante "un poco de aceite para masajear el estómago y luego tiras de la piel de su espalda". Otra participante explicó cómo ella curaba el "empacho" en su casa:

Metiendo soda en el horno y...tu propia saliva...lo colocas alrededor del ombligo y comienzas a frotar, $y$ frotar, $y$ frotar. Entonces te vas a la espalda y comienzas a frotar, y cuando estas frotando levantas la piel de la espalda y tiene que hacer "pum" tres veces, y con eso el "empacho" desaparece.

\section{El susto y su efecto en el hambre y en el sueño de los niños}

Varias madres dijeron que el susto podía provocar que los niños de infantil dejasen de comer y tuvieran problemas con el sueño. Una participante dijo: "El susto les provoca que dejen de comer y ellos se quedan delgados y pierden peso y no quieren comer". Las participantes explicaron que el "susto" ocurría porque los niños se habían llevado una fuerte impresión sobre algo o habían experimentado algo que les había asustado. Una madre explicó lo que provocaba el "susto" en los niños:

Cuando son pequeños y escuchan un sonido fuerte, como el sonido de un coche, una sordina, o como cuando uno grita, eso asusta a un niño. Tienen escalofríos... suele ocurrir que cuando están durmiendo y los levantas, tienen escalofríos y se asustan ...o cuando los despiertas bruscamente.

El "susto" le provocaba al niño que le diera miedo y eso afectaba a su sueño. Una participante describió el comportamiento de sus hijos cuando tenían "susto": "Entonces ellos no pueden dormir. Están todo el rato llorando, sin descansar. Ahí es cuando tú dices, ellos tienen "susto" y tienes que hacerles una "barrida" [barrerles el cuerpo]"

La cura para el "susto" es una "barrida" del cuerpo. La barrida normalmente se hace pasando sobre el cuerpo del niño una rama de árbol "pirul" y rezando una oración. El árbol "pirul" es un tipo de planta que saca toda la mala energía del "susto" (Comunicación personal, Sr. Madrigal-Herbero, febrero 2005).

\section{El mal de ojo y el sueño}

El "mal de ojo" es otra dolencia que las participantes creían que podía afectar el sueño de los niños de edad preescolar. Las participantes explicaron que algunas personas tenían una 
energía muy fuerte que penetraba a través de sus ojos. Una participante afirmó: "mi marido dice que hay un montón de gente [que tiene] una energía o algo parecido en sus ojos". Cuando estas personas se quedan mirando a los niños, las personas pueden transmitir esa fuerte energía a los niños. Los cuerpos de los niños no pueden soportar ese exceso de energía, y no pueden descansar, no pueden dormir y en ocasiones les sube la fiebre. Otra participante explicó qué ocurría cuando una persona con una "mirada fuerte" miraba a tu niño: "la persona de la mirada fuerte tan solo ve [a los niños] y calienta su sangre... [Los niños] justo en ese momento se sienten cansados, no pueden dormir. Esto es debido a que la persona de la mirada fuerte les ha robado el sueño y sus pequeños cuerpos se calientan".

Las participantes creían que la energía fuerte se podía disipar mediante un toque benigno. Cuando las personas sabían que tenían la capacidad de echar un "mal de ojo", ellos tocaban a los niños para disipar la energía acumulada en ellos. En otras ocasiones, cuando la gente decía que los niños de edad preescolar eran bonitos o graciosos, y las madres imaginaban que ellos tenían una energía muy fuerte, podían pedir a otras personas que los tocasen para asegurarse de que el niño no hubiese cogido el mal de ojo. Una madre describió qué pasaría si ella cogiese a su hijo de 4 años en público:

Prestaría atención a las personas, pero como te digo, tú no te das cuenta de la reacción [del mal de ojo] hasta que se van a ir a dormir. Cuando es el momento de irse a la cama es cuando te das cuenta de que su sueño se ha marchado... pero lo que yo haría sería cogerlo y pedirle, "ella es tan guapa, por favor tóquela. Si, tóquela. Mire sus pequeños mofletes. Sí, pero tóquelos".

Las participantes afirmaron que otra forma de prevenir o advertir el "mal de ojo" en los pequeños era colocando un "ojo de venado" sobre los niños cuando fueran a salir en público. El ojo de venado es una semilla redonda, del tamaño aproximado de un cuarto. Es de color marrón fuerte con una gruesa línea oscura que está aproximadamente a tres cuartos del borde de la semilla. Los ojos de venado se venden como parte de un pequeño brazalete bordado con una imagen de un santo en el medio de la semilla. El ojo de venado también se lleva como parte de un alfiler. Una participante afirmó que, tras que su hija de dos años sufriera un episodio de mal de ojo, su marido ahora "se asegura de que ella lleve el brazalete puesto".

Si los niños de preescolar cogen el mal de ojo, la única forma de curarlo es barriendo el cuerpo con un huevo. Las participantes explicaron que se tenía que restregar un huevo sobre el cuerpo del niño mientras se rezaba una oración. Se pensaba que el huevo absorbía la energía que sobraba y la sacaba fuera del niño. Una participante explicó "Digo y creo que un huevo absorbe energía. Esto es lo que pasa, absorbe la energía que le fue transmitida al niño...tan solo paso el huevo sobre la niña y se cura."

El modo en el que las madres sabían que el "mal de ojo" había desaparecido era cuando el niño se dormía rápidamente tras barrer el cuerpo, "ella se duerme automáticamente, en otras palabras, no pasan ni dos minutos, te lo juro, y ella se duerme."

Tras curar a los niños del "mal de ojo", otras madres intentarían proteger el sueño de los niños colocando un par de tijeras abiertas debajo del colchón o colocando una Biblia, un rosario, o una imagen religiosa al lado de la cama del niño. Una participante explicó que las tijeras se usaban como protección: 
Siempre que quieres que no te hagan algo, lo cortas. Cortas lo que se te esté haciendo. [Las tijeras abiertas] representan el símbolo de la cruz. De ese modo, si se le está haciendo algo a ella, imagino que lo cortaría, las tijeras y la cruz lo cortarían.

Para estas participantes, los síndromes culturales de "empacho", "susto" y "mal de ojo" podrían afectar los patrones alimenticios y del sueño de sus hijos, causando de este modo un desequilibrio en el bienestar del niño. Además, las participantes creían que ellas habían sido conscientes de los síndromes culturales que afectaban a la salud de sus hijos y cuidaban esos síndromes mediante el uso de remedios tradicionales para poder recuperar el equilibrio en el bienestar de sus hijos.

\section{DISCUSIÓN}

Este estudio cualitativo ofrece una descripción a fondo de los síndromes culturales y los remedios tradicionales que las madres de ascendencia mexicana usaban para promover y proteger la salud de sus hijos. Los resultados de este estudio son congruentes con los obtenidos en trabajos anteriores ${ }^{(4,5)}$. Sin embargo, los resultados de este estudio llevan más allá la descripción que ofrecen Mikhail y Risser \& Mazur porque ofrece el marco contextual que las madres de ascendencia mexicana usan cuando se enfrentan a los síndromes culturales y los remedios tradicionales. Las participantes de este estudio consideraban que el sueño y una ingesta de comida equilibrada eran importantes para la salud; además, las madres aprovechaban su único conocimiento cultural para curar los síndromes culturales que ellas creían que le impedirían al niño dormir bien o comer de una manera equilibrada. Por otro lado, las madres concebían sus esfuerzos en prevenir el comienzo de estos síndromes culturales como protección de la salud de los niños previniendo las complicaciones y trastornos que pudieran causar.

La manera en la que un síndrome cultural se trata varía de un grupo étnico a otro. Esta diferencia se percibió en la forma en la que se trataba el "mal de ojo" y el "empacho". El "mal de ojo" es un síndrome cultural conocido que existe en varias culturas y grupos étnicos del mundo, pero la forma en la que se trata varía. Tripp-Reimer reveló que los inmigrantes griegos conseguían quitar el mal de ojo bebiendo una mezcla de aceite y agua, haciendo la señal de la cruz sobre el vaso, y luego lavándose la cara con el agua ${ }^{(11)}$. Los remedios tradicionales para el mal de ojo descritos por Tripp-Reimer son diferentes de aquellos usados por las participantes de ascendencia mexicana. El modo en el que se tratan los síndromes culturales puede llegar a variar incluso dentro de la misma etnia. En una muestra similar a la de este estudio, Mikhail encontró que las participantes trataban el "empacho" dándoles a los niños compuestos contaminados de plomo como "azacón" o "greta". "Azacón" y "Greta" son polvos que se mezclan con el agua ${ }^{(4)}$. Sin embargo, las participantes de este estudio trataban el "empacho" masajeando el estómago y la espalda del niño. La diferencia en el tratamiento del "mal de ojo" y del "empacho" probablemente es a causa de las diferencias étnicas y variaciones regionales o geográficas, pero pueden reflejar una necesidad común de dar explicación a los trastornos en las rutinas y los estados de salud de los niños que parece ser que coinciden con otras variaciones en el día a día.

La literatura de los estudios que investigan el uso de la medicina tradicional por personas de ascendencia mexicana ${ }^{(12,13,2)}$ y los resultados encontrados en este estudio señalan que las madres de ascendencia mexicana utilizan a un miembro de la familia o amigos cercanos para que curen los síndromes culturales en lugar de ir a un "curandero".

En el presente estudio, el "empacho", el "susto" y el "mal de ojo" se trataron con la madre del niño. Alguna literatura ha indicado que los padres de ascendencia mexicana van a los 
"curanderos" cuando piensan que sus hijos pueden estar enfermos a causa de algún síndrome cultural, provocando un retraso en el cuidado médico ${ }^{(14)}$. Los resultados en este estudio indican lo contrario. La suposición de que las personas de ascendencia mexicana buscan curanderos cuando hay una enfermedad en lugar de buscar un cuidado médico no se sustenta según los resultados en este estudio. Las madres de ascendencia mexicana en este estudio usaban las fuentes que ellas tenían a su disposición para restablecer la salud de sus hijos. Las participantes llevaban a sus hijos al médico cuando ellas sentían que el cuidado médico curaría a sus hijos. Las participantes usaban remedios culturales para tratar los síndromes culturales porque ellas sabían que un médico especializado en ciencias biomédicas no sabía cómo curar el "empacho", el "susto" o el "mal de ojo". Si los niños no se curaban de estos síndromes culturales, las madres pensaban que un desequilibrio en el cuerpo, mente o alma pondría la salud de los niños en peligro.

Los practicantes deben entender que existen síndromes culturales y que la medicina occidental no tiene una explicación para las causas o les falta el conocimiento para tratarlas. Rechazar los síndromes culturales y sus tratamientos como peyorativos o como superstición es continuar posicionando la medicina occidental en un nivel más alto que las otras formas de sanar. Tener conciencia de los síndromes culturales y de cómo pueden ser percibidos creando una disonancia o desequilibrio en el cuerpo, mente o alma facilitaría la confección de intervenciones culturales específicas en las comunidades de minorías étnicas.

\section{CONCLUSIONES}

\section{Implicaciones para la Educación en Enfermería}

Garantizar que las enfermeras dirijan de manera efectiva las necesidades de cuidados de salud a personas de diversas culturas requiere competencia cultural. Los resultados en este estudio señalan la importancia de la continuada inclusión de la sensibilidad cultural y la apertura en los programas educativos de enfermería. Los profesores deben continuar implementando un currículo que dirija la conciencia y comprensión de los pacientes de diferentes culturas a los estudiantes para garantizar que los futuros enfermeros atiendan de un modo eficiente las necesidades y los cuidados de la salud de los pacientes de ascendencia mexicana y otras culturas. Meleis alegaba que conocer las percepciones de un grupo en particular con respecto a los temas de la salud es esencial para la comprensión que se requiere para desarrollar planes de intervención que mejoren la salud de los pacientes de diversas culturas ${ }^{(15)}$. Los resultados del presente estudio apoyan la continua necesidad de educar a los estudiantes de enfermería en la importancia de ofrecer una sensibilidad cultural en el cuidado de los enfermos.

Las escuelas de enfermería deben incorporan estrategias de enseñanza que faciliten aprender la medicina alternativa complementaria que usan distintas poblaciones. Por ejemplo, una herramienta de enseñanza valiosa sería usar el servicio de proyectos de aprendizaje en enclaves culturales o comunidades donde viven las minorías étnicas. Los estudiantes de enfermería podrían evaluar varios síndromes culturales y las plantas medicinales y remedios tradicionales que usan. Los estudiantes usarían la literatura para evaluar los usos y la seguridad de las hierbas y los remedios tradicionales. De este modo, los estudiantes podrían ofrecer un taller a la comunidad. El taller ofrecería a los miembros de la comunidad las precauciones y recomendaciones relacionadas a una planta medicinal $o$ un remedio tradicional concreto y la información para reforzar síntomas que requieren cuidados alopáticos en lugar de o en adición a los remedios tradicionales. La intención del taller no sería cambiar las creencias de los miembros de la comunidad, sino hacerlos conscientes de los varios usos y precauciones que tienen que tomar cuando usen algunas hierbas medicinales o remedios 
tradicionales en particular. A través del servicio de proyectos de aprendizaje, tales como el del ejemplo presentado, los estudiantes entenderían la lógica subyacente que hay detrás de los síndromes culturales. Esta comprensión suaviza el encuentro enfermero-paciente a un cuidado más efectivo del profesional sanitario.

\section{Implicaciones para la investigación en enfermería}

Los resultados del presente estudio ilustran que comprendiendo cómo las personas de ascendencia mexicana y otros grupos diversos conceptualizan los síndromes culturales que afectan al bienestar de sus hijos puede aumentar la promoción de la salud y del bienestar. La rama de enfermería está al frente de dirigir los estudios para entender los puntos de vista de todo el mundo relacionados con la salud. La investigación futura debería centrarse en explorar y describir cómo otros grupos étnicos definen la promoción y protección de la salud.

\section{Agradecimientos}

El presente trabajo fue financiado por NIH/NINR F31 NR008174. A los autores también les gustaría agradecer al Women's Health Nursing Research Training Grant, NIH/NINR T32 NR0739 y a la Integrating CAM: Nursing Emphasis Grant NIH/NCAM R25 AT001240 por la ayuda en la redacción de éste artículo.

\section{REFERENCIAS}

1. U. S. Census Bureau. 2010 Census Briefs. Overview of Race and Hispanic Origin: 2010. Washington, DC: Population Division, U.S. Census Bureau. Available from: http://www.census.gov/prod/cen2010/briefs/c2010br-02.pdf. 2010.

2. Trotter RT, Chavira JA. Curanderismo-Mexican American folk healing (2 ${ }^{\text {nd }}$ ed.). Athens: University of Georgia Press. 1981.

3. Andrews MM, Boyle JS. Transcultural concepts in nursing care. Philadelphia: Lippincott Williams and Wilkins. 2003.

4. Mikhail BI. Hispanic mother's beliefs and practices regarding selected children's health problems. Western Journal of Nursing Research.1994;16(6):623-638.

5. Risser AL, Mazur JL. Use of folk remedies in a Hispanic population. Archives of Pediatric Adolescent Medicine.1995;149(9):978-981.

6. Gallagher MR., Gill S, Reifsnider E. Child health promotion and protection among Mexican mothers. Western Journal of Nursing Research.2008;30(5):588-605.

7. Spradley JP. The ethnographic interview. New York: Holt, Rinehart and Winston. 1979.

8. Whittemore R, Chase, SK, Mandle, CL. Validity in Qualitative Research. Qualitative Health Research.2001;11(7):522-537.

9. Cuellar I, Arnold B, Maldonado R. Acculturation Rating Scale for Mexican Americans-II: A revision of the original ARSMA scale. Hispanic Journal of Behavioral Sciences.1995;17(3):275-304.

10. Centers for Medicare and Medicaid Services. Who is eligible for Medicaire? Baltimore: Author. Available from: http://www.cms.hhs.gov/medicaid/whoiseligible.asp\#children.2004.

11. Tripp-Reimer T. Retention of folk-healing practices (matiasma) among four generations of urban Greek immigrants. Nursing Research.1983; 32(2):97-101.

12. Chesney AP, Thompson BL, Guevra A, Vela A, Schottstaedt MF. Mexican-American folk medicine: Implications for the family physician. Journal of Family Practice.1980;11(4):567574.

13. Gordon SM. Hispanic cultural health beliefs and folk remedies. Journal of Holistic Nursing. 1994;12(3):307-322. 
14. Flores G. Culture and the patient-physician relationship: Achieving cultural competency in health care. Journal of Pediatrics.2000;36(1):14-23.

15. Meleis Al. Theoretical nursing: Development and progress ( $3^{\text {rd }}$ ed.). New York: Lippincott. 1997. 